\title{
Phenethyl isothiocyanate exhibits antileukemic activity in vitro and in vivo by inactivation of Akt and activation of JNK pathways
}

\author{
N Gao ${ }^{\star, 1,2}$, A Budhraja ${ }^{2}$, S Cheng ${ }^{2}$, E-H Liu ${ }^{1}$, J Chen $^{3}$, Z Yang ${ }^{3}$, D Chen ${ }^{1}$, Z Zhang ${ }^{2}$ and X Shi ${ }^{*, 2}$
}

\begin{abstract}
Effects of phenethyl isothiocyanate (PEITC) have been investigated in human leukemia cells (U937, Jurkat, and HL-60) as well as in primary human acute myeloid leukemia (AML) cells in relation to apoptosis and cell signaling events. Exposure of cells to PEITC resulted in pronounced increase in the activation of caspase- $-3,-8,-9$, cleavage/degradation of PARP, and apoptosis in dose- and time-dependent manners. These events were accompanied by the caspase-independent downregulation of Mcl-1, inactivation of Akt, as well as activation of Jun N-terminal kinase (JNK). Inhibition of PI3K/Akt by LY294002 significantly enhanced PEITC-induced apoptosis. Conversely, enforced activation of Akt by a constitutively active Akt construct markedly abrogated PEITC-mediated JNK activation, Mcl-1 downregulation, caspase activation, and apoptosis, and also interruption of the JNK pathway by pharmacological or genetically (e.g., siRNA) attenuated PEITC-induced apoptosis. Finally, administration of PEITC markedly inhibited tumor growth and induced apoptosis in U937 xenograft model in association with inactivation of Akt, activation of JNK, as well as downregulation of Mcl-1. Taken together, these findings represent a novel mechanism by which agents targeting Akt/JNK/Mcl-1 pathway potentiate PEITC lethality in transformed and primary human leukemia cells and inhibitory activity of tumor growth of U937 xenograft model.
\end{abstract}

Cell Death and Disease (2011) 2, e140; doi:10.1038/cddis.2011.22; published online 7 April 2011

Subject Category: Cancer

Epidemiological studies support the evidence that the consumption of cruciferous vegetables has long been associated with a reduced risk in the occurrence of cancer at various sites, including the prostate, lung, breast, and colon. ${ }^{1}$ The anticarcinogenic effect of cruciferous vegetables is attributed to organic isothiocyanates (ITCs), which are present in a variety of edible cruciferous vegetables such as broccoli, watercress, cabbage, and so on. ${ }^{1}$ Phenethyl isothiocyanate (PEITC) is one of the ITC family of compounds that has attracted a great deal of attention owing to its remarkable cancer chemopreventive activity. ${ }^{2}$ For example, PEITC administration has been shown to significantly inhibit 4-(methylnitrosamino)-1-(3-pyridyl)-1-butanone-induced pulmonary neoplasia in rats and mice, ${ }^{3,4} \mathrm{~N}$-nitrosobenzylmethylamine-induced esophageal cancer in rats, ${ }^{5}$ and benzo[a]pyrene-induced carcinogenesis in mice. ${ }^{6} \mathrm{~A}$ possible mechanism by which PEITC protects against cancer is through cell cycle arrest and apoptosis. ${ }^{7}$ Therefore, an understanding of the mechanism of PEITC-related lethality is important for its further development as a clinically useful chemopreventive or therapeutic agent because this knowledge could lead to the identification of mechanismbased biomarkers potentially useful in future clinical trials.

Recent studies have shown that PEITC is a strong inducer of apoptosis in various cancer cell types. Xiao et al. $^{8}$ have shown that PEITC-induced apoptosis involved extracellular signal-regulated kinase (ERK) activation and the cleavage of procaspase-3, procaspase-8, and procaspase-9 in $\mathrm{PC}-3$ cells. Chen et al. ${ }^{9}$ have reported that apoptosis induction by PEITC is in association with sustained activation of c-Jun $\mathrm{NH} 2$-terminal kinase in Jurkat cells. Huang et al. ${ }^{10}$ provided convincing evidence to suggest an essential role for p53 in apoptosis induction by PEITC. More recent studies have indicated that PEITC-induced cell death involves mitochondria -mediated activation of caspase cascade and generation of reactive oxygen species (ROS). ${ }^{11,12}$ Previous studies have shown that PEITC inhibited growth and induced apoptosis of human leukemia (HL-60) cells, and glutathione metabolism may be involved in PEITC-related lethality. ${ }^{13}$ However, the molecular mechanisms of PEITC-induced apoptosis in human leukemia cells are not fully defined. There is no available information concerning PEITC's in vivo efficacy against

\footnotetext{
${ }^{1}$ Department of Pharmacognosy, College of Pharmacy, III Military Medical University, Chongqing, China; ${ }^{2}$ Graduate Center for Toxicology, College of Medicine, University of Kentucky, Lexington, KY, USA and ${ }^{3}$ Department of Hematology, Southwest Hospital, III Military Medical University, Chongqing, China

${ }^{*}$ Corresponding authors: N Gao, Department of Pharmacognosy, College of Pharmacy, III Military Medical University, Chongqing, China. Tel: + 862368753736 ;

Fax: + 8623 68753046; E-mail: gaoning59@yahoo.com.cn or X Shi, Graduate Center for Toxicology, College of Medicine, University of Kentucky, Lexington, KY 40536-0305, USA. Tel: 859257 4054; Fax: 859323 1059; E-mail: xshi5@email.uky.edu

Keywords: phenethyl isothiocyanate; Akt; JNK; apoptosis; leukemia; xenograft

Abbreviations: AML, acute myeloid leukemia; Bad, Bcl-2 associated agonist of cell death; Bax, Bcl-2 associated X protein; Bcl-2, B- cell lymphoma; ERK, extracellular signal-regulated kinase; FITC, fluorescein isothiocyanate; GSK, glycogen synthase kinase; H\&E, hematoxylin and eosin; JNK, Jun N-terminal kinase; MAPK, mitogenactivated protein kinase; Mcl-1, myeloid cell leukemia sequence 1; mTOR, mammalian target of rapamycin; NOD/SCID, non-obese diabetic/severe combined immunodeficiency; PARP, poly ADP-ribose polymerase; PEITC, phenethyl isothiocyanate; PI, propidium iodide; PI3K, phosphoinositide-3-kinase; PTEN, phosphatase and tensin homolog; TUNEL, terminal deoxynucleotidyl transferase dUTP nick end labeling; UV, ultraviolet; XIAP, X-linked inhibitor of apoptosis protein Received 08.2.11; revised 23.2.11; accepted 23.2.11; Edited by A Stephanou
} 
leukemia. This study provides experimental evidence to indicate, for the first time, that the cell death caused by PEITC is initiated by the inactivation of Akt, leading, in turn, to Jun N-terminal kinase (JNK) activation, and culminating in $\mathrm{Mcl}-1$ downregulation. In addition, we show that administration of PEITC significantly inhibits the tumor growth of U937 xenografts in SCID mice in association with inactivation of Akt, activation of JNK, as well as induction of apoptosis.

\section{Results}

PEITC induces apoptosis, caspase activation, and PARP cleavage in U937 human leukemia cells in dose- and time-dependent manners. A dose-dependent study of U937 cells exposed to various concentrations of PEITC for 3 and $6 \mathrm{~h}$ was shown in Figure 1a; modest degrees of apoptosis were noted at $4 \mu \mathrm{M}$ PEITC concentration, which increased substantially at concentration of $6 \mu \mathrm{M}$. These events became very extensive at concentration of $8 \mu \mathrm{M}$. A time-course study of cells exposed to $8 \mu \mathrm{M}$ PEITC revealed a significant increase in apoptosis as early as $3 \mathrm{~h}$, and reached near-maximal levels after $6 \mathrm{~h}$ of drug exposure (Figure $1 \mathrm{~b}$ ).

Consistent with these results, exposure of cells to $4 \mu \mathrm{M}$ PEITC for 3 and $6 \mathrm{~h}$ revealed a moderate increase in cleavage/activation of caspase-3, -8 , and -9 , as well as PARP degradation and a marked increase at concentrations $\geq 6 \mu \mathrm{M}$ (Figure 1c). Time-course study of cells exposed to $8 \mu \mathrm{M}$ a

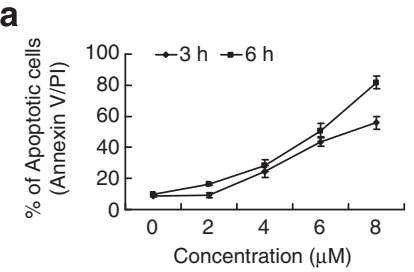

C

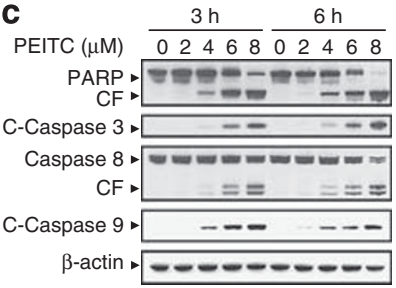

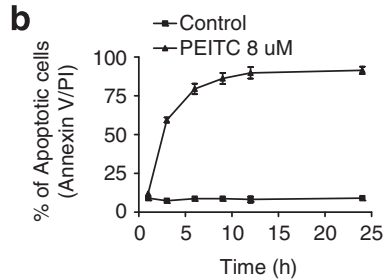

d

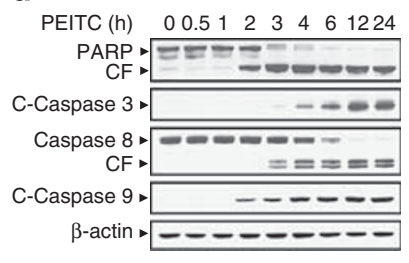

Figure 1 Phenethyl isothiocyanate (PEITC) significantly induces apoptosis, caspase activation, and poly(ADP-ribose) polymerase (PARP) cleavage in U937 human leukemia cells in dose- and time-dependent manners. (a) U937 cells were treated without or with various concentrations of PEITC as indicated for 3 and $6 \mathrm{~h}$. (b) U937cell were treated without or with $8 \mu \mathrm{M}$ PEITC for $1,3,6,9,12$, and $24 \mathrm{~h}$. Cells were stained with Annexin V/propidium iodide (PI), and apoptosis was determined using flow cytometry as described in Materials and Methods. (c) U937 cells were treated without or with various concentrations of PEITC as indicated for 3 and $6 \mathrm{~h}$. (d) U937 cells were treated without or with $8 \mu \mathrm{M}$ PEITC for $0.5,1,2,3,4,6$, 12 , and $24 \mathrm{~h}$. After treatment of U937 cells with the indicated PEITC concentration or the indicated time interval, total cellular extracts were prepared and subjected to western blot assay using antibodies against PARP, cleaved-caspase-3 (C-Caspase-3), caspase-8, and cleaved-caspase-9 (C-Caspase-9). Each lane was loaded with $30 \mu \mathrm{g}$ protein. Blots were subsequently stripped and reprobed with antibody against $\beta$-actin to ensure equivalent loading and transfer. Two additional studies yielded equivalent results
PEITC revealed moderate increases in cleavage/activation of caspase-3, -8 , and -9 , as well as PARP degradation 2 or $3 \mathrm{~h}$ after drug exposure. These events became apparent at $4 \mathrm{~h}$, and reached near-maximal levels at $6 \mathrm{~h}$ after drug exposure (Figure 1d).

Exposure of U937 cells to PEITC results in rapid decrease in $\mathrm{Mcl}-1$ protein level. To understand the critical roles of $\mathrm{Bcl}-2$ family proteins have in apoptosis, expression of these proteins in U937 cells was monitored following treatment with PEITC. As shown in Figure 2a, exposure of cells to $4 \mu \mathrm{M}$ PEITC for 3 and $6 \mathrm{~h}$ resulted in moderate decrease in the expression of $\mathrm{Mcl}-1$. These events became apparent at $6 \mu \mathrm{M}$ and very extensive at $8 \mu \mathrm{M}$ after drug exposure. A time-course study showed that exposure of U937 cells to $8 \mu \mathrm{M}$ PEITC resulted in modest decrease in the expression of $\mathrm{Mcl}-1$ as early as $2 \mathrm{~h}$ after drug exposure. These events became apparent at $3 \mathrm{~h}$ and very extensive at $4 \mathrm{~h}$ after drug exposure (Figure 2b). In contrast, the expression of other Bcl-2 family proteins such as XIAP, Bcl-2, $\mathrm{Bcl}-\mathrm{xL}$, Bax, and Bad remained unaffected (Figures $2 \mathrm{a}$ and b). Taken together, these findings show that downregulation of Mcl-1 may be responsible for PEITC lethality in U937 cells.

Exposure of U937 cells to PEITC results in marked decrease in levels of phospho-Akt and significant increase in the expression of phospho-JNK. Effects of PEITC on various signal-transduction pathways implicated in apoptosis regulation were also investigated.
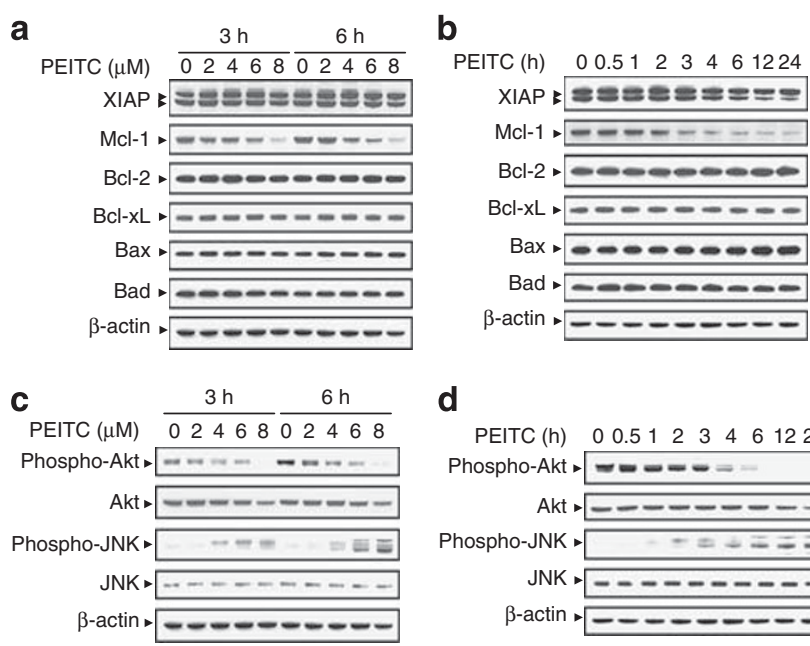

d

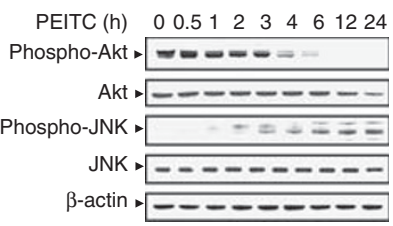

Figure 2 Phenethyl isothiocyanate (PEITC) induces downregulation of Mcl-1, inactivation of Akt, and activation of Jun N-terminal kinase (JNK) in U937 cells. (a) U937 cells were treated without or with the indicated concentrations of PEITC for 3 and $6 \mathrm{~h}$. (b) U937 cells were treated without or with $8 \mu \mathrm{M}$ PEITC for $0.5,1,2,3,4$, 6,12 , and $24 \mathrm{~h}$. Total cellular extract were prepared and subjected to western blot analysis using antibody against XIAP, Mcl-1, Bcl-2, Bcl-xL, Bax, and Bad. (c) U937 cells were treated without or with the indicated concentrations of PEITC for 3 and $6 \mathrm{~h}$. (d) U937 cells were treated without or with $8 \mu \mathrm{M}$ PEITC for $0.5,1,2,3,4,6,12$, and $24 \mathrm{~h}$. Total cellular extract were prepared and subjected to western blot analysis using antibodies against cell signaling molecules, including phospho-Akt, Akt, phospho-JNK, and JNK. Each lane was loaded with $30 \mu \mathrm{g}$ protein. Blots were subsequently stripped and reprobed with antibody against $\beta$-actin to ensure equivalent loading and transfer. Two additional studies yielded equivalent results 
Dose-dependent study indicated that exposure of U937 cells to PEITC at $2 \mu \mathrm{M}$ resulted in a slight reduction in levels of phospho-Akt (Ser473). These events became apparent at concentration of 4 and $6 \mu \mathrm{M}$. The complete blockage of levels of phospho-Akt (Ser473) was noted after drug exposure at $8 \mu \mathrm{M}$ (Figure 2c). However, PEITC had little or no significant effects on total Akt. In addition, treatment of cells with PEITC resulted in a dramatic increase in levels of phospho-JNK, but had no effect on levels of total JNK. A time-course study showed that exposure of U937 cells to $8 \mu \mathrm{M}$ PEITC resulted in modest decrease in levels of phospho-Akt and a increase in the activation of JNK as early as $2 \mathrm{~h}$ after drug exposure. These events became apparent at 4 and $6 \mathrm{~h}$ and reached near-maximal effects at 12 or $24 \mathrm{~h}$ (Figure 2d). In contrast, PEITC had little or no effect on expression of total or phospho-mTOR, phospho-ERK, and phospho-p38 (data not shown). These results suggest that a pronounced inactivation of Akt and an activation of JNK may have important roles in PEITC-induced apoptosis in U937 human leukemia cells.

PEITC has similar effects on apoptosis in multiple human leukemia cell types and primary AML blasts. Various human leukemia cell types, including U937 myelomonocytic, HL-60 promyelocytic, and Jurkat T-lymphoblastic leukemic cells, were exposed to $8 \mu \mathrm{M}$ PEITC for $6 \mathrm{~h}$, after which apoptosis was determined by Annexin V/PI analysis. As shown in Figure $3 a$, treatment with PEITC resulted in dramatic increase in apoptosis in these cell lines.
Consistent with these results, exposure of cells to PEITC revealed significant increases in cleavage/activation of caspase-3, -8, and -9 , as well as PARP degradation (Figure 3b). Similarly, exposure of various human leukemia cells to PEITC resulted in marked decrease in expression of Mcl-1 (Figure 3c). Comparable inactivation of Akt and activation of JNK were also observed in these cell lines after drug exposure (Figure 3d).

Furthermore, exposure of blasts from 17 patients with acute myeloid leukemia (AML) to $8 \mu \mathrm{M}$ PEITC for $24 \mathrm{~h}$ revealed marked increase in cell death (Figure $3 \mathrm{e}$ ), accompanied by cleavage/activation of caspase-3, -8 , and -9 , as well as PARP degradation (Figure $3 f$ ). In contrast, the PEITC regimen exerted only minimal toxicity toward normal human peripheral blood mononuclear cells (Figure $3 \mathrm{~g}$ ). Taken together, these findings indicate that PEITC selectively triggers apoptosis in multiple human leukemia cells lines as well as in primary AML blasts, but displays significantly less toxicity toward normal hematopoietic cells.

PEITC lethality is associated with the caspaseindependent inactivation of Akt and activation of JNK. To assess whether PEITC-induced inactivation of Akt and activation of JNK are dependent on caspases activation, the pan-caspase inhibitor Z-VAD-FMK was used. Cotreatment of cells with the caspase inhibitor Z-VAD-FMK, which abrogated PEITC-induced apoptosis (Figure 4a), caspase activation, and PARP degradation (Figure 4b), failed to prevent Akt inactivation, JNK activation, as well as a

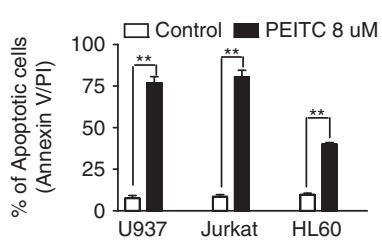

b

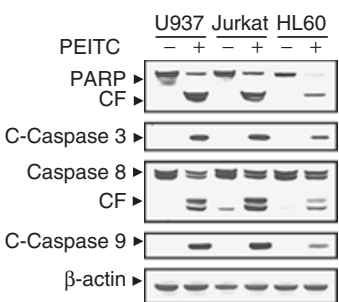

f
C

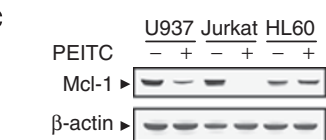

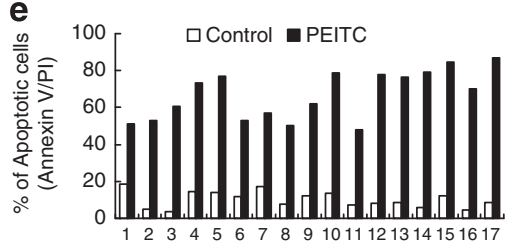


a
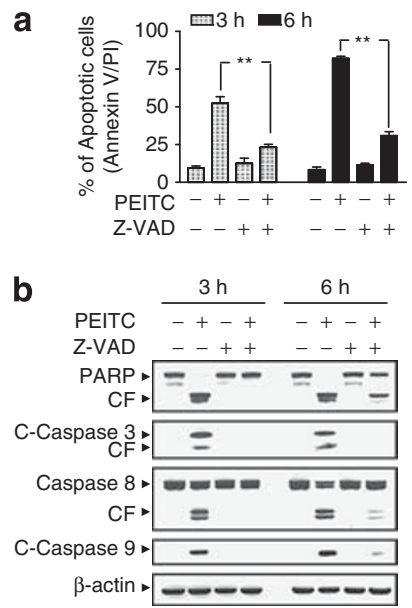

C

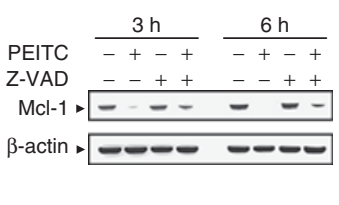

d

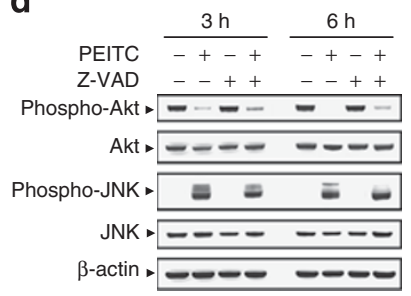

Figure 4 Phenethyl isothiocyanate (PEITC)-induced Mcl-1 downregulation, Akt inactivation, and Jun N-terminal kinase (JNK) activation were not dependent on caspase activation. U937 cells were pretreated with the caspase inhibitor benzyloxycarbonyl-Val-Ala-Asp(O-methyl)-fluoromethylketone (Z-VADFMK) $(10 \mu \mathrm{M})$ for $1 \mathrm{~h}$, followed by treatment with $8 \mu \mathrm{M}$ of PEITC for 3 and $6 \mathrm{~h}$. (a) Cells were stained with Annexin V/propidium iodide (PI). Apoptosis was determined using flow cytometry as described in Materials and Methods. The values obtained from Annexin $V$ assays represent the means \pm standard deviation (S.D.) for three separate experiment. ${ }^{* *}$ Values for cells treated with PEITC and Z-VADFMK were significantly reduced compared with values obtained for PEITC alone by Student's $t$-test; $P<0.01$. Total protein extracts were prepared and subjected to western blot assay using antibodies against apoptosis-related proteins, including PARP, cleaved-caspase-3 (C-Caspase-3), caspase-8, and C-Caspase-9 (b); antiapoptotic protein Mcl-1 (c); as well as cell signaling proteins, including phospho-Akt, Akt, phospho-JNK, and JNK (d). For western blot analysis, each lane was loaded with $30 \mu \mathrm{g}$ of protein, and blots were subsequently stripped and reprobed with antibody against $\beta$-actin to ensure equivalent loading

Mcl-1 downregulation induced by PEITC (Figures 4c and d). Such findings indicate that inactivation of Akt, activation of JNK, and downregulation of Mcl-1 mediated by PEITC do not simply represent a secondary, caspase-dependent event.

The effects of interruption of PI3K/Akt signaling pathway on PEITC-induced apoptosis. To assess the effects of interruption of the PI3K/Akt pathway on the lethal actions of PEITC, U937 cells were exposed to sublethal concentration of PEITC (i.e., $4 \mu \mathrm{M}$; 25\%) in the presence of PI3K inhibitor LY294002 (i.e., $20 \mu \mathrm{M}$ ), after which apoptosis was assessed. As shown in Figure 5a, co-administration of LY294002 with PEITC resulted in a pronounced increase apoptosis in U937 cells $(P<0.01$ versus PEITC treatment alone). Similarly, co-administration of PEITC and LY294002 at concentrations that were ineffective or marginally effective by themselves resulted in pronounced increase in the activation of caspase3 , -8, and -9 , and PARP degradation (Figure 5b). Combined treatment with PEITC and LY294002 also resulted in the potentiation of $\mathrm{Mcl}-1$ downregulation (Figure $5 \mathrm{c}$ ). Furthermore, co-administration of PEITC and LY294002 essentially abrogated expression of the phosphorylated Akt (Ser473) and potentiated activation of JNK (Figure 5d). Because PEITC inhibits phosphorylation of Akt and functions like Akt inhibitor, we used $4 \mu \mathrm{M}$ PEITC instead of $8 \mu \mathrm{M}$.
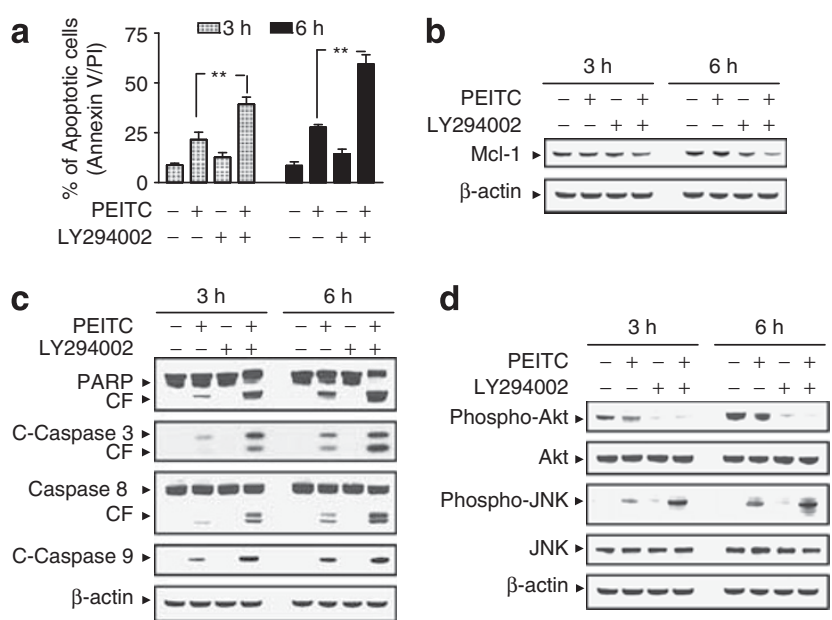

Figure 5 Inhibition of phosphoinositide 3-kinase (PI3K)/Akt by LY294002 (LY) enhanced phenethyl isothiocyanate (PEITC)-induced apoptosis in human leukemia cells. U937 cells were pretreated with $20 \mu \mathrm{M}$ of $\mathrm{LY}$ for $1 \mathrm{~h}$, followed by the addition of $4 \mu \mathrm{M}$ of PEITC for 3 and $6 \mathrm{~h}$. (a) Cells were stained with Annexin V/propidium iodide (PI), and apoptosis was determined using flow cytometry as described in Materials and Methods. The values obtained from Annexin V/PI assays represent the means \pm standard deviation (S.D.) for three separate experiments. ${ }^{*} V$ Values for cells treated with PEITC and LY in combination were significantly greater than those for cells treated with PEITC alone by Student's $t$-test; $P<0.01$. Total cellular extracts were prepared as described in Materials and Methods, and subjected to western blot analysis using antibodies against apoptosis-related proteins, including poly(ADP-ribose) polymerase (PARP), cleaved-caspase-3 (C-Caspase-3), caspase-8, and C-Caspase-9 (b), antiapoptotic protein Mcl-1 (c), and cell signaling molecules, including phospho-Akt, Akt, phospho-Jun N-terminal kinase (JNK), and JNK (d). For western blot assay, each lane was loaded with $30 \mu \mathrm{g}$ of protein; blots were subsequently stripped and reprobed with antibody against $\beta$-actin to ensure equivalent loading. Two additional studies yielded equivalent results

To characterize the functional role of perturbations in Akt in the apoptotic response of leukemia cells to PEITC, U937 cells ectopically expressing constitutive active form of Akt (Akt-CA) and the dominant-negative Akt (Akt-DN) were employed. As shown in Figure 6a, exposure of pcDNA3.1 vector control cells and Akt-DN cells to PEITC at a concentration of $8 \mu \mathrm{M}$ for $6 \mathrm{~h}$ resulted in significant increase in apoptosis. However, apoptosis was strikingly attenuated in Akt constitutively active cells after drug exposure. Consistent with these results, the activation of caspases and cleavage of PARP were also markedly attenuated in Akt-CA cells (Figure 6b). In addition, enforced activation of Akt efficaciously blocked PEITCmediated Mcl-1 downregulation (Figure 6c). Western blot analysis displayed marked increase in levels of total and phospho-Akt in Akt-CA cells. PEITC failed to inhibit phosphorylation of Akt in these Akt cells (Figure 6d). Interestingly, the ability of PEITC to induce JNK activation was essentially abrogated in Akt-CA cells (Figure 6d). Taken together, these findings indicate that inactivation of the Akt pathway has a critical role in PEITC-related lethality and that this event lies upstream of Mcl-1 downregulation and JNK activation.

Effects of pharmacological and genetic inhibition of JNK on PEITC-related lethality. The functional role of JNK activation in PEITC lethality was then investigated using both pharmacological and genetic approaches. As shown in 


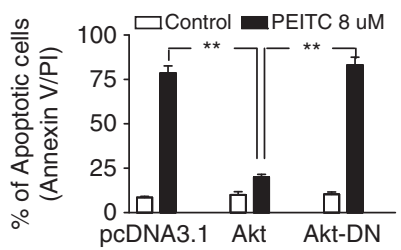

c

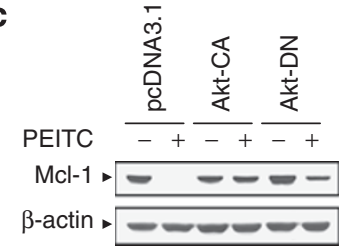

b

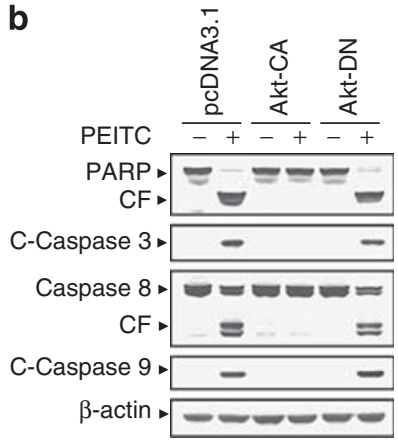

d

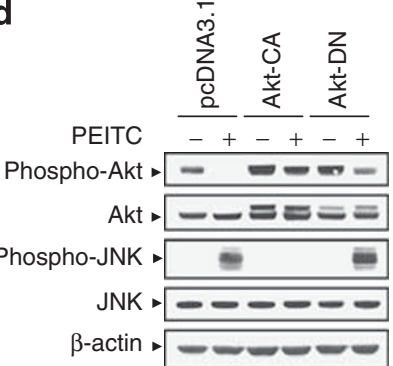

Figure 6 Enforced activation of Akt markedly protects cells from phenethyl isothiocyanate (PEITC)-induced apoptosis. U937 cells were stably transfected with constitutively active forms of Akt (Akt-CA), dominant-negative Akt mutant (Akt-DN), and an empty vector (pcDNA3.1) as described in Materials and Methods. All cells were then treated with $8 \mu \mathrm{M}$ of PEITC for $6 \mathrm{~h}$. (a) After treatment, apoptosis was determined using Annexin V- fluorescein isothiocyanate (FITC) assay as described in Materials and Methods. ${ }^{* *}$ Values for Akt-CA cells treated with PEITC were significantly decreased compared with those for pcDNA3.1 and Akt-DN cells by Student's $t$-test; $P<0.01$. Total cellular extracts were prepared and subjected to western blot analysis using antibodies against apoptosis-related proteins, including poly(ADP-ribose) polymerase (PARP), cleaved-caspase-3 (C-Caspase-3), caspase-8, and C-Caspase-9 (b), antiapoptotic protein Mcl-1 (c), and cell signaling proteins, including phospho-Akt, Akt, phospho-Jun N-terminal kinase (JNK), and JNK (d). For western blot assay, each lane was loaded with $30 \mu \mathrm{g}$ of protein; blots were subsequently stripped and reprobed with antibody against $\beta$-actin to ensure equivalent loading. Two additional studies yielded equivalent results

Figure $7 \mathrm{a}$, co-administration of a non-toxic concentration of SP600125 (i.e., $10 \mu \mathrm{M}$ ) with a toxic concentration of PEITC $(8 \mu \mathrm{M} ; \sim 75 \%$ for $6 \mathrm{~h})$ resulted in a pronounced decrease in apoptosis (i.e., to $\sim 30 \%$ ). Consistently, co-administration of SP600125 also markedly attenuated PEITC-mediated caspases activation and PARP degradation (Figure 7b). Co-administration of SP600125 also attenuated PEITCinduced Mcl-1 downregulation and JNK activation (Figures 7c and d). As SP600125 is not completely specific for JNK, ${ }^{14}$ a genetic approach utilizing JNK1 siRNA was employed. As shown in Figure $7 f$, transient transfection of U937 cells with JNK1 siRNA reduced the expression of JNK1 to $50 \%$ compared with control cells, and resulted in a significant reduction in PEITC-mediated apoptosis $(P<0.01$ versus control siRNA cells). Taken together, these findings indicate that JNK activation has an important functional role in PEITC-related lethality.

PEITC inhibits tumor growth of U937 xenograft model accompanied by striking induction of apoptosis, inactivation of Akt, and activation of JNK. To assess whether our in vitro observations could be translated into an a

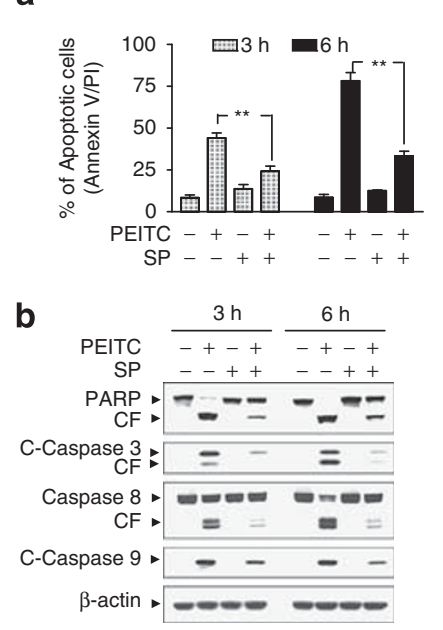

c

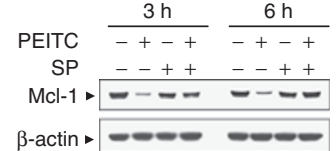

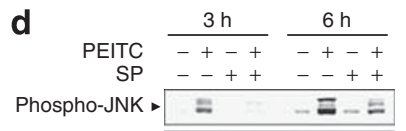

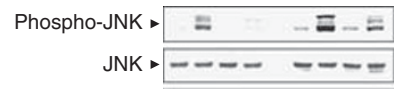

$\beta$-actin $-\infty-\infty-\infty$

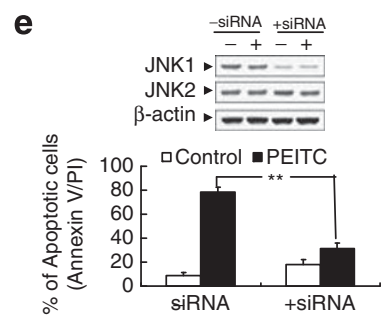

Figure 7 Effects of pharmacological and genetic interruption of Jun N-terminal kinase (JNK) on phenethyl isothiocyanate (PEITC)-induced apoptosis. U937 cells were pretreated with $10 \mu \mathrm{M}$ of JNK inhibitor, SP600125 (SP), for $1 \mathrm{~h}$, followed by the addition of $8 \mu \mathrm{M}$ of PEITC for 3 and $6 \mathrm{~h}$. (a) Cells were stained with Annexin V/PI, and apoptosis was determined using flow cytometry as described in Materials and Methods. The values obtained from Annexin V/PI assays represent the means \pm standard deviation (S.D.) for three separate experiments. ${ }^{*} V$ Values for cells treated with PEITC and SP were significantly less than those obtained for cells treated with PEITC alone by Student's $t$-test; $P<0.01$. After treatment, total cellular extracts were prepared and subjected to western blot analysis using antibodies against poly(ADP-ribose) polymerase (PARP), cleaved-caspase-3 (C-Caspase-3), caspase-8, and C-Caspase-9 (b), Mcl-1 (c), as well as cell signaling proteins, including phospho-JNK, and JNK (d). (e) U937 cells were transiently transfected with JNK1 small interfering RNA (siRNA) oligonucleotides or controls and incubated for $24 \mathrm{~h}$ at $37^{\circ} \mathrm{C}$, after which cells were treated with $8 \mu \mathrm{M}$ of PEITC for $6 \mathrm{~h}$. Total cellular extracts were prepared and subjected to western blot analysis using antibody against JNK1 and JNK2. Apoptosis was determined using the Annexin V-FITC assay as described in Materials and Methods. **Values for cells treated with PEITC after transfection with JNK1 siRNA oligonucleotides were significantly decreased compared with those for control cells treated with PEITC by Student's t-test; $P<0.01$

animal model system, NOD/SCID mice were inoculated intraperitoneally (i.p.) with U937 cells, after which mice received injections with vehicle or PEITC (50 mg/kg, i.p.) for 20 days starting 3 days after the injection of U937 human leukemia cells. As shown in Figure $8 \mathrm{a}$, treatment with PEITC resulted in a modest, but significant suppression of tumor growth 10 days following drug exposure $(P<0.05$ versus vehicle control). These events became more apparent 15 and 20 days after drug exposure $(P<0.01$ between PEITC treatment and vehicle control). In contrast, no statistically significant change in body weight was noted comparing vehicle control and PEITC regimen (Figure $8 b$ ). Moreover, the mice of PEITC group did not exhibit any other signs of toxicity such as agitation, impaired movement and posture, indigestion or diarrhea, and areas of redness or swelling. These results indicated that PEITC administration significantly inhibited tumor growth of U937 xenograft without causing any side effects and/or toxicity to the mice.

The morphological changes and induction of apoptosis in U937 cells in vivo were investigated using hematoxylin and eosin (H\&E) staining and TUNEL assay. As shown in 
a

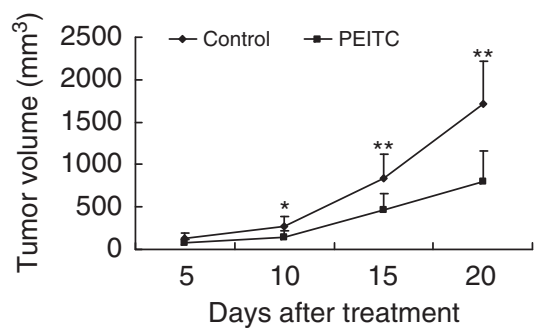

c

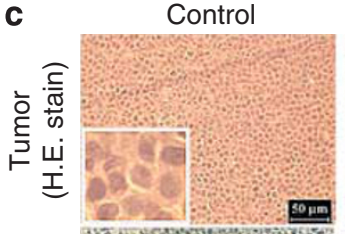

Control

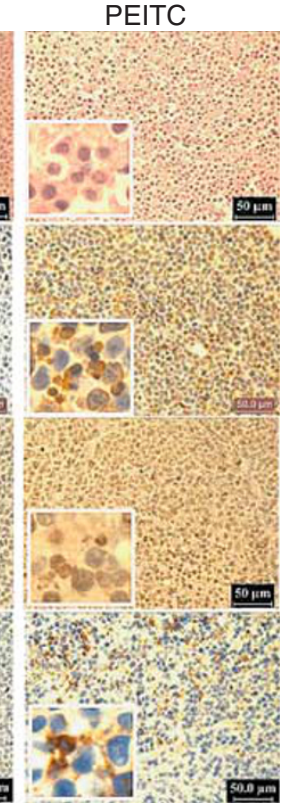

b

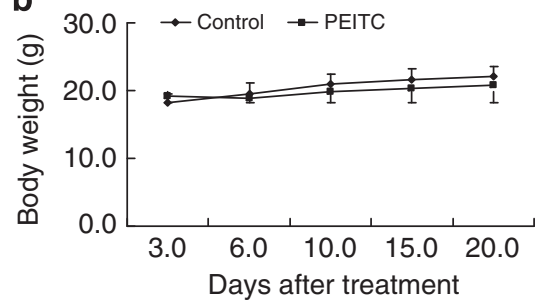

d

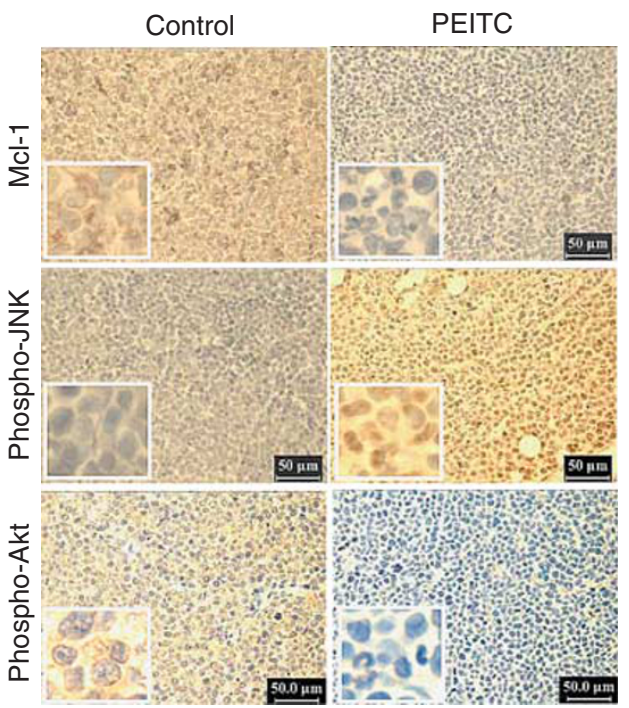

Figure 8 Phenethyl isothiocyanate (PEITC) inhibits tumor growth and induces apoptosis in U937 xenograft animal model. Twenty non-obese diabetic/severe combined immunodeficiency (NOD/SCID) mice were inoculated with U937 cells $\left(2 \times 10^{6}\right.$ cells per mouse, subcutaneously (s.c.)) and randomly divided into two groups (10 per group) for treatment with PEITC (50 mg/kg, intraperitoneally (i.p.), daily) or with vehicle control solvent as described in Materials and Methods. (a) Average tumor volume in vehicle control mice and mice treated with $50 \mathrm{mg} / \mathrm{kg}$ PEITC. Data are means \pm standard deviation (S.D.) (10 mice per group with tumors implanted on right flank of each mouse). * or **Values for tumor volume of U937 xenograft mice treated with PEITC were significantly decreased compared with vehicle control by Student's $t$-test; $P<0.05$ or $P<0.01$. (b) Body weight changes of mice during the 20 days of treatment. Statistical analysis of body weight changes showed no significant differences between PEITC treatment and vehicle control groups. (c) Tumors were removed from animals 20 days after the start of drug exposure. Tumors were fixed and stained with hematoxylin and eosin (H\&E) stain to examine tumor cell morphology, using terminal deoxynucleotidyl transferase dUTP nick-end labeling (TUNEL) assay to determine apoptosis, and using immunohistochemistry to determine the levels of cleaved-poly(ADP-ribose) polymerase (C-PARP) and cleaved-caspase-3 (C-Caspase-3). (d) After treatment with PEITC for 20 days, tumor tissues were also sectioned and subjected to immunohistochemistry using antibodies against Mcl-1, phospho-Akt, and phospho-Jun N-terminal kinase (JNK). The sections were lightly counterstained with hematoxylin and photographed with a Scan Scope. Original magnification $\times 400$

Figure 8c, the sections of U937 xenografts from mice treated with PEITC exhibited a reduced number of cancer cells, with signs of necrosis with infiltration of inflammatory cells (i.e., phagocytic cells), fibrosis, as well as apoptotic regions, identified by their amorphous shape and condensed nuclei. Moreover, exposure to PEITC resulted in a striking induction of apoptosis in tumor cells, with signs of numerous dark brown-colored apoptotic cells. Also, exposure to PEITC caused a rapid increase in immunoreactivity for the cleaved form of PARP and caspase-3, indicative of apoptosis.

The preceding findings implied that downregulation of Mcl-1, inactivation of Akt, and activation of JNK might have important roles in PEITC-mediated lethality in U937 cells in vitro. To evaluate whether the preceding in vitro findings would be operative in vivo, immunohistochemistry analysis was performed to evaluate the expression of $\mathrm{Mcl}-1$, phosphoAkt, and phospho-JNK in tissue sections of U937 xenografts.
As shown in Figure 8d, immunostaining of tumors from mice treated with PEITC showed that the levels of $\mathrm{Mcl}-1$ and phospho-Akt were decreased and phospho-JNK was increased. Collectively, these findings suggest that disruption of Akt/JNK/Mcl-1 signaling may also contribute to lethality induced by PEITC in vivo.

\section{Discussion}

These results indicate that PEITC markedly induces cell death in multiple human leukemia cell types and primary human AML blast cells. Our results also provide detailed molecular mechanistic information as to how PEITC exerts its apoptotic effects on human leukemia cells (i.e., by inactivation of Akt, activation of JNK, and downregulation of $\mathrm{Mcl}-1$ ). Previous studies indicated that PEITC was shown to induce apoptosis 
in many cancer cells such as prostate,${ }^{8,11}$ breast, ${ }^{15}$ lung, ${ }^{16}$ hepatoma, ${ }^{17}$ colon, ${ }^{18}$ as well as leukemia. ${ }^{13}$ Mechanism of PEITC-induced apoptosis is not fully understood, but known cellular responses to this promising agent include $\mathrm{ERK}{ }^{8}$ $\mathrm{JNK}^{9}{ }^{9}$ nuclear factor- $\kappa \mathrm{B}(\mathrm{NF}-\kappa \mathrm{B}),{ }^{19}$ and ROS-mediated apoptosis induction. ${ }^{11,12}$ Presently, little information is available concerning the functional role of the Akt pathway in mediating PEITC-induced lethality, particularly in malignant hematopoietic cells. The results of this study show that Akt inactivation not only represents the primary cause, but also have an important functional contribution in JNK activation, Mcl-1 downregulation, and lethality induced by PEITC.

Akt is a serine-threonine kinase intimately involved in the regulation of cell survival. ${ }^{20}$ It is activated by recruitment to the cell membrane through the actions of PI3K, which in turn is regulated by the PTEN phosphatase ${ }^{21}$ mutations of which are among the most commonly encountered in human cancers. ${ }^{22}$ Akt is involved in activation/phosphorylation of numerous downstream targets implicated in the modulation of diverse pathways governing cell survival decisions, including those associated with glycogen synthase kinase (GSK), ${ }^{23} \mathrm{~m}$-TOR/p70rsk, ${ }^{24}$ and NF$\kappa \mathrm{B},{ }^{25}$ among others. Most notably, PEITC exposure resulted in diminished, rather than increased, Akt phosphorylation. Although it would be tempting to attribute this phenomenon to PTEN activation, the fact that U937 cells do not express wild-type PTEN argue against this notion. ${ }^{26} \mathrm{~A}$ more likely possibility is that PEITC, through a mechanism yet to be elucidated, blocks the actions of PI3K. The finding that LY29004, an inhibitor of PI3K, enhanced the lethality of PEITC by blocking the activation of Akt and one or more of its downstream targets (i.e., Mcl-1) is potentially consistent with this concept.

In fact, the caspase-dependent downregulation of Akt is a well-described phenomenon. ${ }^{27}$ Our present results indicate that PEITC induces apoptosis by activating both caspase-8 and -9 , along with caspase-3, raising the possibility that Akt inactivation might represent a consequence of engagement of the caspase cascade. In this study, co-treatment of U937 cells with the pan-caspase inhibitor Z-VAD-FMK, which abrogated PEITC-induced activation of caspases (i.e., caspase-3, -8, and -9) and apoptosis, failed to prevent Akt inactivation, arguing strongly that factors other than caspase-mediated events are involved in this phenomenon.

The bulk of evidence suggests that in human leukemia cells, PEITC-induced Akt inactivation has a critical functional role in mediating PEITC lethality. Significantly, enforced activation of Akt and prevention of PEITC-induced Akt downregulation largely reversed the lethal consequences of PEITC exposure, including caspase activation, PARP cleavage, and apoptosis. It is worth noting that PEITC exposure resulted in the downregulation of $\mathrm{Mcl}-1$, an antiapoptotic protein that may play a particularly important role in regulating apoptosis in malignant hematopoietic cells. ${ }^{28}$ It has been reported that the antiapoptotic gene $\mathrm{Mcl}-1$ is upregulated by the PI3K/Akt signaling pathway, ${ }^{29}$ and downregulation of $\mathrm{Mcl}-1$ by inhibition of PI3K/Akt pathway is required for cell death. ${ }^{30}$ The finding that enforced activation of Akt largely blocked PEITC-mediated downregulation of $\mathrm{Mcl}-1$ may significantly contribute to PEITC-mediated lethality.

Induction of caspase activation and apoptosis by PEITC was also associated with the activation of the stress-related
JNK pathway. JNK belongs to the superfamily of MAP kinases that are involved in the regulation of cell proliferation, differentiation, and apoptosis. ${ }^{31}$ The critical role of JNK has been shown in the lethal effects of diverse cytotoxic stimuli, including ceramide, ${ }^{32}$ Fas ligand, ${ }^{33} \mathrm{UV},{ }^{34}$ among others. The finding that pharmacological and genetic interruption of the JNK pathway attenuated PEITC-mediated lethality indicates that stress pathways have a critical functional role in apoptosis induction by this agent. Interestingly, co-administration with PI3K inhibitor LY294002, which potentiate inactivation of Akt, enhanced JNK activation and apoptosis induced by PEITC. Furthermore, enforced activation of Akt not only blocked PEITC-mediated caspase activation and apoptosis, but also prevented the striking increase in JNK activation, raising the possibility that one of the mechanisms by which Akt protects cells from PEITC lethality is by opposing JNK activation. This phenomenon might be explained by the following lines of evidence. Firstly, ASK-1, the protein that activates JNK, is a target of Akt inhibitory phosphorylation. Phosphorylation by Akt inhibited JNK activity, which is mediated by ASK1, providing the direct link between Akt and JNK. ${ }^{20}$ Secondly, the interaction between Akt and JIP1 inhibited JIP1-mediated potentiation of JNK activity by decreasing JIP1 binding to specific JNK pathway kinases, suggesting that Akt interaction with JIP1 acts as a negative switch for JNK activation. ${ }^{35}$

Previous studies have shown that PEITC markedly inhibits tumor growth of human prostate and ovarian xenografts through the induction of apoptosis. ${ }^{11,12,36,37}$ Little is known, however, about inhibitory effects of PEITC on tumor growth of human leukemia xenograft model. The results from in vivo studies have shown that PEITC exhibits significant inhibitory effects on the growth of U937 leukemia tumor xenograft. To understand whether the apoptotic mechanism in vitro is identical to those in vivo, we next examined apoptosis in tumor specimens obtained from control and PEITC-treated animals using TUNEL staining. The increase of TUNEL-positive cells was detected in the PEITC-treated group compared with the control group, which provides the apoptotic evidence in PEITC-treated U937 xenograft mice. This is the first report that describes an effective extrapolation of the in vitro apoptosis-inducing effects of PEITC on the leukemia cells to the in vivo situation. We also found that administration of PEITC resulted in marked increase in activation/cleavage of caspase-3 and PARP in tissue sections of U937 tumor xenograft, further confirming the apoptotic mechanism observed in vivo. To dissect the possible mechanisms in which the apoptotic signaling was triggered, we also examined the expression levels of phospho-Akt, phospho-JNK, and Mcl-1 in tissue sections of U937 xenograft tumor using immunohistochemistry analysis. Our results suggest that suppression of the p-Akt and Mcl-1 and upregulation of p-JNK were closely correlated with the reduction of U937 tumor xenografts. These results are consistent with the in vitro data. In addition, the lack of toxicity in a mouse model points to PEITC as a potential candidate for chemopreventive and chemotherapeutic use. These data suggest that PEITC can be a possible anticancer agent in the treatment of leukemia and possibly other hematological malignancies.

In summary, these findings indicate that PEITC effectively induces cell death in human leukemia cells, including primary leukemia blasts, as well as in leukemia xenografts. This effect 
occurs in association with the inactivation of Akt, activation of JNK, as well as downregulation of Mcl-1, such findings could have chemopreventive or chemotherapeutic implications.

\section{Materials and Methods}

Cell lines. U937, HL-60, and Jurkat cells were purchased from American Type Culture Collection (ATCC, Manassas, VA, USA) and maintained in RPMI 1640 medium containing $10 \%$ fetal bovine serum (FBS). The Akt-CA, which is an Myc-tagged myr-Akt, and the Akt-DN were kindly provided by Dr. Richard Roth (Stanford University, School of Medicine, Stanford, CA, USA), and were subcloned into the pcDNA3.1. U937 cells were stably transfected with Akt-CA and Akt-DN using the Amaxa nucleofector (Koeln, Germany) as recommended by the manufacturer. Stable single-cell clones were selected in the presence of $400 \mu \mathrm{g} /$ $\mathrm{ml}$ of geneticin. Thereafter, the expression of Akt from each cell clone was analyzed by western blot analysis.

Peripheral blood samples for the in vitro studies were obtained from 17 patients with newly diagnosed or recurrent AML after informed consent. Approval was obtained from the Southwest Hospital (Chongqing, China) institutional review board for these studies. AML blasts were isolated by density gradient centrifugation over Histopaque-1077 (Sigma Diagnostics, St. Louis, MO, USA) at $400 \times \mathrm{g}$ for $38 \mathrm{~min}$. Isolated mononuclear cells were washed and assayed for total number and viability using Trypan blue exclusion. Blasts were suspended at $8 \times 10^{5} / \mathrm{ml}$ and incubated in RPMl 1640 medium containing 10\% FBS in 24-well plates. Fresh normal peripheral blood mononuclear cells were purchased from Allcells (Emeryvill, CA, USA). After washing and enumerating, cells were suspended at $8 \times 10^{5} / \mathrm{ml}$ before treatment.

Reagents and antibodies. PEITC was purchased from LKT Laboratories (St. Paul, NM, USA). LY294002, SP600125, and Z-VAD-FMK were purchased from EMD Biosciences (La Jolla, CA, USA). Antibodies against Akt, JNK, phospho-JNK, and $\beta$-actin were from Santa Cruz Biotechnology (Santa Cruz, CA, USA); cleaved caspase-3, cleaved caspase-9, phospho-Akt (Ser473), and Bcl-xL were from Cell Signaling Technology (Beverly, MA, USA); XIAP, Mcl-1, Bax, and Bad were from BD PharMingen; PARP was from Biomol (Plymouth Meeting, PA, USA); caspase-8 was from Alexis (Carlsbad, CA, USA) and Bcl-2 was from Dako (Carpinteria, CA, USA).

RNA interference and transfection. U937 cells $\left(1.5 \times 10^{6}\right)$ were transfected with $1 \mu \mathrm{g}$ JNK1-annealed dsRNAi oligonucleotide $5^{\prime}$-CGUGGGAU UUAUGGUCUGUGTT-3'/3'-TTGCACCUAAAUACCAGACAC-5' (Orbigen, San Diego, CA, USA) using the Amaxa nucleofectort (Koeln, Germany) as recommended by the manufacturer. After incubation at $37^{\circ} \mathrm{C}$ for $24 \mathrm{~h}$, transfected cells were treated with PEITC, and subjected to determination of apoptosis and JNK expression using Annexin V/PI and flow cytometry and western blot analysis as described below.

Apoptosis. The extent of apoptosis in leukemia cells was evaluated by flow cytometric analysis using FITC-conjugated Annexin V/propidium iodide (BD PharMingen) staining as per the manufacturer's instructions as described previously. ${ }^{38}$ Both early apoptotic (Annexin V-positive, PI-negative) and late apoptotic (Annexin V-positive and PI-positive) cells were included in cell death determinations.

Western blot analysis. The total cellular samples were washed twice with ice-cold PBS and lysed in $1 \times$ NuPAGE LDS sample buffer supplemented with $50 \mathrm{mM}$ dithiothreitol. The protein concentration was determined using Coomassie Protein Assay Reagent (Pierce, Rockford, IL, USA). In total, $30 \mu \mathrm{g}$ of sample proteins were separated by SDS-PAGE and transferred to nitrocellulose membrane. Membranes were blocked with $5 \%$ fat-free dry milk in $1 \times$ Tris-buffered saline (TBS) and incubated with antibodies. Protein bands were detected by incubating with horseradish peroxidase-conjugated antibodies (Kirkegaard and Perry Laboratories, Gaithersburg, MD, USA) and visualized with enhanced chemiluminescence reagent (Perkin-Elmer, Boston, MA, USA).

Xenograft. NOD/SCID mice (5 weeks old) were purchased from Vital River Laboratories (Beijing, China). All animal studies were conducted according to protocols approved by the Institutional Animal Care and Use Committee of the University. U937 cells $\left(2 \times 10^{6} / 0.2 \mathrm{ml}\right.$ per mouse) were suspended in sterile PBS and injected subcutaneously into the right flank of the mice. Mice were randomized into two groups of 10 mice per group. At 3 days after tumor inoculation, the treatment group received PEITC (50 mg/kg, i.p. for 20 days). The control group received an equal volume of solvent control. Tumor size and body weight were measured after treatment at various time intervals throughout the study. At the termination of the experiment, mice were killed at $24 \mathrm{~h}$ after the last administration of compound. The tumors were excised and weighed. Tumors were collected at selected times and fixed in paraformaldehyde. Paraffin-embedded tissues were sectioned and processed for H\&E, TUNEL, and immunohistochemical staining.

TUNEL. The apoptotic cells in tissue samples were detected using an In Situ Cell Death Detection kit (Roche, Mannheim, Germany) according to the manufacturer's manual. After deparaffinization and permeabilization, the tissue sections were incubated in proteinase $\mathrm{K}$ for $15 \mathrm{~min}$ at room temperature. The sections were then incubated with the TUNEL reaction mixture that contains terminal deoxynucleotidyl transferase and fluorescein-dUTP at $37^{\circ} \mathrm{C}$ for $1 \mathrm{~h}$. After washing three times with PBS, the sections were incubated with the ConverterPOD, which contains anti-fluorescein antibody conjugated with horse-radish peroxidase (POD) at room temperature for $30 \mathrm{~min}$. After washing three times with PBS, the sections were incubated with $0.05 \% \quad 3-3^{\prime}$-diaminobenzidine tetrahydrochloride and analyzed under light microscope.

Histological and immunohistochemical evaluation. At the termination of experiments, tumor tissues from representative mice were sectioned, embedded in paraffin, and stained with H\&E for histopathological evaluation. For immunohistochemical analysis, tissue sections $4 \mu \mathrm{m}$ in thickness were dewaxed and rehydrated in xylene and graded alcohols. Antigen retrieval was performed with $0.01 \mathrm{M}$ citrate buffer at $\mathrm{pH} 6.0$ for $20 \mathrm{~min}$ in a $95^{\circ} \mathrm{C}$ water bath. Slides were allowed to cool for another $20 \mathrm{~min}$, followed by sequential rinsing in PBS and TBS-T buffer. Endogenous peroxidase activity was quenched by incubation in TBS-T containing 3\% hydrogen peroxide. Each incubation step was carried out at room temperature and was followed by three sequential washes ( 5 min each) in TBS-T. After blocking with $10 \%$ goat serum for $1 \mathrm{~h}$, sections were incubated with primary antibodies, washed three times in PBS, incubated with biotinylated secondary antibody for $1 \mathrm{~h}$, followed by incubation with a streptavidin-peroxidase complex for another $1 \mathrm{~h}$. After three additional washes in PBS, diaminobenzidine working solution was applied. Finally, the slides were counterstained in hematoxylin.

Statistical analysis. Tumor volumes, body weights, and percentage of apoptotic cells were represented as mean \pm S.D. The statistical significance of the difference between control and PEITC-treated groups was evaluated using Student's $t$-test. $P<0.05$ or 0.01 were considered significant.

\section{Conflict of Interest}

The authors declare no conflict of interest.

Acknowledgements. This study was supported by Grant Number RO1 ES015375 (X Shi) from the National Institute of Health (NIH).

1. Conaway CC, Yang YM, Chung FL. Isothiocyanates as cancer chemopreventive agents: their biological activities and metabolism in rodents and humans. Curr Drug Metab 2002; 3 : 233-255.

2. Stoner GD, Morrissey DT, Heur YH, Daniel EM, Galati AJ, Wagner SA. Inhibitory effects of phenethyl isothiocyanate on $\mathrm{N}$-nitrosobenzylmethylamine carcinogenesis in the rat esophagus. Cancer Res 1991; 51: 2063-2068.

3. Morse MA, Wang CX, Stoner GD, Mandal S, Conran PB, Amin SG et al. Inhibition of 4-(methylnitrosamino)-1-(3-pyridyl)-1-butanone-induced DNA adduct formation and tumorigenicity in the lung of F344 rats by dietary phenethyl isothiocyanate. Cancer Res 1989; 49: 549-553.

4. Morse MA, Amin SG, Hecht SS, Chung FL. Effects of aromatic isothiocyanates on tumorigenicity, 06-methylguanine formation, and metabolism of the tobacco-specific nitrosamine 4-(methylnitrosamino)-1-(3-pyridyl)-1-butanone in A/J mouse lung. Cancer Res 1989; 49: 2894-2897.

5. Stoner GD, Morrissey DT, Heur YH, Daniel EM, Galati AJ, Wagner SA. Inhibitory effects of phenethyl isothiocyanate on $\mathrm{N}$-nitrosobenzylmethylamine carcinogenesis in the rat esophagus. Cancer Res 1991; 51: 2063-2068.

6. Hecht SS, Kenney PM, Wang M, Trushin N, Upadhyaya P. Effects of phenethyl isothiocyanate and benzyl isothiocyanate, individually and in combination, on lung tumorigenesis induced in A/J mice by benzo[a]pyrene and 4-(methylnitrosamino)-1-(3pyridyl)-1-butanone. Cancer Lett 2000; 150: 49-56. 
7. Xiao D, Zeng Y, Choi S, Lew KL, Nelson JB, Singh SV. Caspase-dependent apoptosis induction by phenethyl isothiocyanate, a cruciferous vegetable-derived cancer chemopreventive agent, is mediated by Bak and Bax. Clin Cancer Res 2005; 11: 2670-2679.

8. Xiao D, Singh SV. Phenethyl isothiocyanate-induced apoptosis in p53-deficient PC-3 human prostate cancer cell line is mediated by extracellular signal-regulated kinases. Cancer Res 2002; 62: 3615-3619.

9. Chen YR, Wang W, Kong AN, Tan TH. Molecular mechanisms of c-Jun N-terminal kinasemediated apoptosis induced by anticarcinogenic isothiocyanates. J Biol Chem 1998; 273: 1769-1775.

10. Huang C, Ma WY, Li J, Hecht SS, Dong Z. Essential role of p53 in phenethyl isothiocyanate-induced apoptosis. Cancer Res 1998; 58: 4102-4106.

11. Xiao D, Lew KL, Zeng Y, Xiao H, Marynowski SW, Dhir R et al. Phenethyl isothiocyanateinduced apoptosis in PC-3 human prostate cancer cells is mediated by reactive oxygen species-dependent disruption of the mitochondrial membrane potential. Carcinogenesis 2006; 27: 2223-2234.

12. Trachootham D, Zhou Y, Zhang H, Demizu Y, Chen Z, Pelicano H et al. Selective killing of oncogenically transformed cells through a ROS-mediated mechanism by $b$-phenylethyl isothiocyanate. Cancer Cell 2006; 10: 241-252.

13. Xu K, Thornalley PJ. Involvement of GSH metabolism in the cytotoxicity of the phenethyl isothiocyanate and its cysteine conjugate to human leukaemia cells in vitro. Biochem Pharmacol 2001; 61: 165-177.

14. Minutoli L, Altavilla D, Marini H, Passaniti M, Bitto A, Seminara P et al. Protective effects of SP600125 a new inhibitor of c-jun N-terminal kinase (JNK) and extracellular-regulated kinase (ERK1/2) in an experimental model of cerulein-induced pancreatitis. Life Sci 2004; 75: 2853-2866.

15. Lee JW, Cho MK. Phenethyl isothiocyanate induced apoptosis via down regulation of $\mathrm{Bcl}$ 2/XIAP and triggering of the mitochondrial pathway in MCF-7 Cells. Arch Pharm Res 2008; 31: 1604-1612.

16. Yang YM, Jhanwar-Uniyal M, Schwartz J, Conaway CC, Halicka HD, Traganos F et al $\mathrm{N}$-acetylcysteine conjugate of phenethyl isothiocyanate enhances apoptosis in growthstimulated human lung cells. Cancer Res 2005; 65: 8538-8547.

17. Wu SJ, Teik Ng L, Lin CC. Effects of antioxidants and caspase-3 inhibitor on the phenylethyl isothiocyanate-induced apoptotic signaling pathways in human PLC/PRF/5 cells. Eur J Pharmacol 2005; 518: 96-106.

18. Bonnesen C, Eggleston IM, Hayes JD. Dietary indoles and isothiocyanates that are generated from cruciferous vegetables can both stimulate apoptosis and confer protection against DNA damage in human colon cell lines. Cancer Res 2001; 61 6120-6130.

19. Xu C, Shen G, Chen C, Gélinas C, Kong AN. Suppression of NF-kappaB and NF-kappaBregulated gene expression by sulforaphane and PEITC through IkappaBalpha, IKK pathway in human prostate cancer PC-3 cells. Oncogene 2005; 24: 4486-4495.

20. Kim AH, Khursigara G, Sun X, Franke TF, Chao MV. Akt phosphorylates and negatively regulates apoptosis signal-regulating kinase 1. Mol Cell Biol 2001; 21: 893-901.

21. Cantley LC, Neel BG. New insights into tumor suppression: PTEN suppresses tumor formation by restraining the phosphoinositide 3-kinase/AKT pathway. Proc Natl Acad Sci USA 1999; 96: 4240-4245.

22. Mao JH, To MD, Perez-Losada J, Wu D, Del Rosario R, Balmain A. Mutually exclusive mutations of the Pten and ras pathways in skin tumor progression. Genes Dev 2004; 18 $1800-1805$
23. Pap M, Cooper GM. Role of glycogen synthase kinase-3 in the phosphatidylinositol 3-kinase/Akt cell survival pathway. J Biol Chem 1998; 273: 19929-19932.

24. Edinger AL, Thompson CB. Akt maintains cell size and survival by increasing mTORdependent nutrient uptake. Mol Biol Cell 2002; 13: 2276-2288.

25. Gelfanov VM, Burgess GS, Litz-Jackson S, King AJ, Marshall MS, Nakshatri H et al. Transformation of interleukin-3-dependent cells without participation of Stat5/bcl-xL: cooperation of akt with raf/erk leads to $\mathrm{p} 65$ nuclear factor $\kappa \mathrm{B}$-mediated antiapoptosis involving C-IAP2. Blood 2001; 98: 2508-2517.

26. Liu TC, Lin PM, Chang JG, Lee JP, Chen TP, Lin SF. Mutation analysis of PTEN/MMAC1 in acute myeloid leukemia. Am J Hematol 2000; 63: 170-175.

27. Yang JY, Widmann $\mathrm{C}$. The RasGAP N-terminal fragment generated by caspase cleavage protects cells in a Ras/PI3K/Akt-dependent manner that does not rely on NF-kappaB activation. J Biol Chem 2002; 277: 14641-14646.

28. Rinkenberger JL, Horning S, Klocke B, Roth K, Korsmeyer SJ. Mcl-1 deficiency results in peri-implantation embryonic lethality. Genes Dev 2000; 14: 23-27.

29. Wang JM, Chao JR, Chen W. The antiapoptotic gene mcl-1 is up-regulated by the phosphatidylinositol 3-kinase/Akt signaling pathway through a transcription factor complex containing CREB. Mol Cell Biol 1999; 19: 6195-6206.

30. Araki T, Hayashi M, Watanabe N, Kanuka H, Yoshino J, Miura M et al. Down-regulation of Mcl-1 by inhibition of the PI3-K/Akt pathway is required for cell shrinkage-dependent cell death. Biochem Biophys Res Commun 2002; 290: 1275-1281.

31. Dhanasekaran DN, Reddy EB. JNK signaling in apoptosis. Oncogene 2008; 27: 6245-6251.

32. Verheij M, Bose R, Lin XH, Yao B, Jarvis WD, Grant S et al. Requirement for ceramideinitiated SAPK/JNK signalling in stressinduced apoptosis. Nature 1996; 380: 75-79.

33. Wilson DJ, Fortner KA, Lynch DH, Mattingly RR, Macara IG, Posada JA et al. JNK, but not MAPK, activation is associated with Fas-mediated apoptosis in human T cells. Eur $J$ Immunol 1996; 26: 989-994.

34. Zanke BW, Boudreau K, Rubie E, Winnett E, Tibbles LA, Zon L et al. The stress-activated protein kinase pathway mediates cell death following injury induced by cis-platinum, UV irradiation or heat. Curr Biol 1996; 6: 606-613.

35. Kim AH, Yano H, Cho H, Meyer D, Monks B, Margolis B et al. Akt1 regulates a JNK scaffold during excitotoxic apoptosis. Neuron 2002; 35: 697-709.

36. Khor TO, Keum YS, Lin W, Kim JH, Hu R, Shen G et al. Combined inhibitory effects of curcumin and phenethyl isothiocyanate on the growth of human PC-3 prostate xenografts in immunodeficient mice. Cancer Res 2006; 66: 613-621.

37. Bommareddy A, Hahm ER, Xiao D, Powolny AA, Fisher AL, Jiang Y et al. Atg5 regulates phenethyl isothiocyanate-induced autophagic and apoptotic cell death in human prostate cancer cells. Cancer Res 2009; 69: 3704-3712.

38. Gao N, Budhraja A, Cheng S, Yao H, Zhang Z, Shi X. Induction of apoptosis in human leukemia cells by grape seed extract occurs via activation of c-Jun NH2-terminal kinase. Clin Cancer Res 2009; 15: 140-149.

Cell Death and Disease is an open-access journal published by Nature Publishing Group. This work is licensed under the Creative Commons Attribution-Noncommercial-No Derivative Works 3.0 Unported License. To view a copy of this license, visit http://creativecommons.org/licenses/by-nc-nd/3.0/ 